\title{
Lost and Found, Letters and Methods: Assessing Attitudes toward Chiropractic and Medical Care
}

\author{
Hannah Kern \\ Florida State University
}

\author{
William H. Yeaton \\ Tallahassee, FL
}

Attitudes toward traditional and chiropractic medicine were compared using Milgram's lost letter technique. A total of 192 letters were placed on the windshields of vehicles in parking lots at six restaurants and department stores in each of four quadrants of a medium-sized, Southeastern city. These letters were addressed to "Admissions" at either a fictitious Institute of Medicine or Institute of Chiropractic Care. Return addresses included either a male or a female name. Thus, those who found a lost letter were faced with the option of returning or not returning a letter from either a male or a female, addressed to an Institute of traditional or non-traditional medicine. After examining previous studies which had used the lost letter technique, numerous methodological improvements were implemented. For example, letters were randomly assigned to potential drop spots for each of 24 study locations (six study locations in each of four city quadrants), and a Latin square design was used to control for possible order effects in the four study conditions that were implemented. Nearly $65 \%$ of the letters (124 of 192) were returned. We found: 1) letters addressed to a fictitious Institute of Chiropractic Care were just as likely to be returned as those addressed to a fictitious Institute of Medicine; 2) letters with female return addresses were as likely to be returned as those with male return addresses; 3) there was no interaction between study conditions; 4) based on what was essentially a replication study, a comparison of the pattern of returns using the first and second cycle of lost letters $(n=96$ for each cycle) revealed an equivalent pattern of no-difference findings.

Milgram (1977) created the lost letter (LL) technique to minimize the demand characteristics and social desirability pressures germane to written questionnaires (Webb, Campbell, Schwartz, \& Sechrest, 1966). The method has been widely used to assess attitudes toward presidential candidates (Wicker, 1969), to determine attitudes toward the Vietnam War (Berkowitz, 1970), to assess attitudes toward socially undesirable groups (Milgram, Mann, \& Harter, 1965), and to evaluate the acceptance of controversial medical procedures such as abortion (Kunz \& Fernquist, 1989). Recently, the method has been expanded to include lost e-mails in addition to paper mail (Bushman \& Bonacci, 2004). Ironically, though introduced several decades ago, lost letter methods can sometimes provide a useful antidote for the current and growing trend in psychology to base findings on self-report rather than actual behavior (Baumeister, Vohs, \& Funder, 2007).

One area in which LL methods might be particularly relevant is consumer sentiment regarding health care options. Attitudes toward medical services appear to be changing and various modalities of complementary and alternative medicine (CAM) are now well documented as a substantial source of care in the United States (Eisenberg, Kessler, Foster, Norlock, Calkins, \& Delbanco, 1993). In particular, utilization of chiropractic services has substantially increased in recent years (Kessler, 2001; Lawrence \& Meeker, 2007). This secular trend toward greater acceptance of non-traditional medicine, when coupled with consumer's increasing disillusion with more traditional forms of medicine (Beard, 2004), suggests that the gap in our sentiments toward these two approaches has narrowed. However, one might also argue that when consumers are asked to make a direct choice, traditional medical approaches are still preferred.

In the current study, LL was used to compare attitudes toward chiropractic care and traditional medicine. Data were gathered relatively soon after a controversial decision to 


\section{KERN AND YEATON}

abandon the creation of a post-graduate training program in chiropractic medicine at a large, local University. This controversy was fueled by substantial Legislative dollars earmarked to fund the new Program, which were subsequently cancelled, effectively ending the plan. Considerable media attention in newspapers and on the radio had been paid to this divisive issue (e.g., Matus, 2004).

\section{Methods in Prior LL Studies}

The social science, researcher community has long accepted the LL technique as a powerful method for ascertaining attitudes without relying on self-reports and their inherent weaknesses (e.g., Orne, 1962). However, after reviewing numerous published instances of the implementation of LL, we found a variety of methodological weaknesses. Prominent researchers in the social sciences have long urged more careful attention to high quality research design features (Shadish, Cook, \& Campbell, 2002; Shadish \& Cook, 2009). Thus, the major aim of this study was to improve upon these methodological shortcomings.

Often, prior research using LL has not clearly articulated procedures used to drop letters. For example, letters were sometimes left in unspecified locations within phone booths or on the ground near entrances and exits to buildings with no apparent rule regarding precise placement. Finally, choice of specific locations within communities for placing letters seemed arbitrary, though malls and phone booths were quite common.

These procedures lead to several conceptual problems. For example, when a letter is dropped "on the ground," many persons may see the lost letter, pass by but ignore it, or pick it up but choose not to mail it, or pick the letter up and put it in the mail. If the tenth person who had seen a lost letter chooses to pick it up and mail it, should the return rate be $10 \%$ or $100 \%$ ? In this example, exposure to the lost letter becomes an uncontrolled variable that ultimately makes murky the estimate of return rates. A procedure ensuring that a specific individual who sees a letter has to make a decision regarding its return represents a significant methodological improvement.

The most serious flaw in previous implementations of LL is the sometimes incomplete, often under-described, and usually unsystematic set of procedures used in the placement of letters. In perhaps the first, published lost-letter study, Milgram and his colleagues allocated 400 letters to four conditions (1965). The author's note: "The letters were systematically distributed in 10 districts of the city and in four types of placements... Twenty five envelopes with each address were distributed in each placement." (p. 437)

Here, all four of the study conditions were equally present in each of the four types of placements (e.g., phone booths). However, the manner in which the four equal sets of 100 envelopes were distributed to the 10 districts was not specified, leaving the possibility that between-district differences were related to return rate differences.

Subsequent applications of the LL methodology are lacking in other ways. For example, Wicker (1969), who used the LL technique to assess preference for a Presidential candidate, describes the procedures as follows:

“...letters were dropped in four locations: street pavements, stores, telephone booths, and under automobile windshield wipers... In each Ward, $15 \mathrm{E}$ and $15 \mathrm{C}$ letters were dropped at each of the four types of locations." (p. 260)

In this instance, we do not know how the $\mathrm{E}$ and $\mathrm{C}$ letters were allocated to the four kinds of locations. Were the majority of letters dropped at stores rather than on street pavements? For an individual store, were both $\mathrm{E}$ and $\mathrm{C}$ letters dropped? And might there be systematic differences in social economic status (SES) between the four types of locations? 


\section{REFINING LOST LETTER METHODS}

In yet another example, Simmons and Zumpf (1983), placed 30 letters for each of seven study conditions and noted that: "The letters were dropped in shopping center stores... or placed under the windshields of cars parked in shopping center lots." (p. 512)

An astute reader wonders if the description is simply incomplete or if the authors gave little thought to the way in which the letters were dropped. Thus, when a specific location was chosen, that location did not necessarily receive all study conditions, and the order in which conditions were implemented was not clearly specified. Also, the study location was potentially confounded by condition, and this major shortcoming allowed the possibility that the particular demographics and SES of a location contributed to spurious and unreliable differences in return rates. Unfortunately, previous studies have not consistently reported within-city return rates by location, making it difficult to estimate the impact of SES. In the case of the present study, greater care was given to account for a location's potential impact on attitude because opinions toward both conventional and CAM have been shown to be related to SES (Conboy, Kaptchuk, Eisenberg, Gottlieb, \& Acevedo-Garcia, 2007).

Clearly, such studies place individual letters within a given location unsystematically and, perhaps, based on the judged convenience of the research staff. Such a methodological error is suspiciously similar to an analogous culprit in quota sampling; when researchers have discretion over those who are included in a survey, systematic bias often occurs (e.g., Freedman, Pisani, \& Purves, 1997), which produces demonstrably incorrect estimates of voting preference.

We used several tactics to correct these weaknesses: Each study location received all of the study conditions with the order of receipt based on a Latin Square design; a relatively large number of locations were sampled from all areas of the city; specific automobiles were randomly sampled from within the parking lot of each location; and letters were placed under the windshield wiper of each targeted vehicle.

\section{Research Hypotheses}

As noted above, although recent evidence suggests an increasingly favorable view regarding alternative medical approaches, consumer's attitudes toward traditional medicine remains generally more favorable than toward alternative medical approaches such as chiropractic (e.g., Gould, 2005). In addition, the research evidence indicates that females are substantially more likely to receive help than males (cf., Eagly \& Crowley, 1986). Finally, studies of sex role stereotyping (Eccles, 1986) generally indicate that consumers believe that males tend to gravitate to positions of greater responsibility (e.g., physicians) while females choose those of lesser authority (e.g, nurses). Thus, in the context of this research, we hypothesized that:

Hypothesis 1. Ho: Prop IOM > Prop ICC. More letters would be returned that were addressed to an Institute of Medicine (IOM) than to an Institute of Chiropractic Care (ICC);

Hypothesis 2. Ho: Prop F > Prop M. More letters from females (F) would be returned than letters from males $(\mathrm{M})$;

Hypothesis 3. Ho: Institute $\mathrm{x}$ Gender $=0$. There would be a significant interaction between the kind of care and gender; return rates would be higher for females in the chiropractic condition and males in the medical condition but lower for males in the chiropractic condition and females in the medical condition. 


\section{Method}

\section{Placement of Lost Letters}

A single stamped letter was placed stamp-side up, under the windshield wiper, on the driver's side, of an automobile randomly chosen from parking lots in a medium sized city in the Southeastern United States. Either a co-author or one of two research assistants inconspicuously carried each letter to the target vehicle. Upon reaching an automobile, the person dropping the single letter bent down as if picking it up from the ground then placed the letter under the windshield wiper. Borrowing a tactic from previous research (Milgram et al., 1965), we included a handwritten note with each lost letter stating "I found this next to your car." Each note was written on one of four, equal-sized, ripped sections of a white, $81 / 2$ " by 11 " sheet of paper.

Locations and Drops. The city was divided into four areas based on the intersection of two major streets running through the downtown. Although each quadrant reflected racial and economic homogeneity, there was considerable heterogeneity across the quadrants. We chose six locations within each quadrant: two fast food restaurants (e.g., McDonalds), two "sit down" restaurants (e.g., Fridays), and two locations that included either a single or many, large department stores. These businesses covered the entire geography within each quadrant; plus, no two businesses were in close proximity. Two additional locations were added to the original list of 24 sites, once because the restaurant was closed and once because there were fewer than two eligible automobiles (defined below) in the parking lot.

Ninety-six letters were distributed on each of two, different weekend days (Saturday from 11AM to 3 PM and Sunday from 3 PM to 7 PM), four weeks apart. On each of the two days, two letters were dropped by four different researchers, at each of the six locations, in each of the four quadrants (a total of $n=48$ per quadrant). There were two cycles within each drop day; for cycle one, each researcher distributed two letters at each of six locations $(n=24)$; then, approximately two hours later, each researcher exactly repeated the procedure, for the same locations (another $\mathrm{n}=24$ ). Upon return for the second cycle, if a letter was still present on an auto's windshield at a given location, the auto was ineligible for a drop. (See Figure 1 for a visual representation of the study design.)

\begin{tabular}{|llcccc|}
\hline & & Q1 (R1) & Q2 (R2) & Q3 (R3) & Q4 (R4) \\
& Location & L1-6 & L1-6 & L1-6 & L1-6 \\
\hline Weekend 1 & & & & & \\
& Cycle 1 & C & B & D & A \\
& Cycle 2 & D & A & B & C \\
\hline Weekend 2 & & & & & \\
& Cycle 1 & B & C & A & D \\
& Cycle 2 & A & D & C & B \\
\hline
\end{tabular}

Figure 1. Schematic of the lost letter research design. Two letters are "lost" within each of the 96 cells of the LL design; Q 1-4 = Quadrants of city; R 1-4 = Researchers; L 1-6 = Locations within a quadrant; A, B, C, D = Conditions of the study, ordered using the Latin square design; Four conditions = male-medical, female-medical, male-chiropractic, female-chiropractic. 


\section{REFINING LOST LETTER METHODS}

Selection of Automobiles. We conceptualized a parking lot as having one or more rectangular grids with each parking space in a unique row (r) and column (c). By estimating the total number of parking spaces within each rectangle ( $\mathrm{r}$ times $\mathrm{c}$ ) and adding these totals, we obtained an estimate of the maximum number of parked cars in each lot. Then, using a random number table, each researcher identified, at each location, two target autos for each cycle and day. If a target vehicle was not eligible ("broken down," commercial, and those vehicles without license plates indicating the local county were omitted), or if the randomly chosen parking space was unoccupied, we chose the first eligible auto to the right of the target vehicle. Occupied vehicles or autos being approached by an apparent owner were omitted. If it was raining, or if rain was forecast, the scheduled drop day was postponed.

\section{Study Conditions}

Each return address was typed so that half designated an ethnically neutral male name (Jason), while the other half designated an ethnically neutral female name (Jessica). All letters had the home address of one researcher (WHY), where letters were subsequently returned if mailed by a finder. Half the envelopes were addressed to the "Institute of Chiropractic Care" and half to the "Institute of Medicine" (for purposes of the study, neither Institute exists). The Institute name appeared in bold letters, separated by a single line from the rest of the typed address. On the second line of the address, the words "Attention: Admissions" were included followed by a fictitious box number. Each envelope contained a brief message asking the Institute to confirm the applicant's admission status. All information within each letter was double-checked by an author to confirm consistency with the specific condition indicated on the envelope.

One of 24 letters of the alphabet (excluding X and Z) was placed in the position of the middle initial of the sender's return address, and this letter uniquely identified each of the 24 locations. A period after the middle initial of the return addressee designated all letters dropped in the first cycle of a given drop day; the absence of a period designated a second-cycle drop. There were 48 letters in each of the four conditions of the study: male-medical; male-chiropractic; femalemedical; and female-chiropractic (the letters A-D in the Latin square design below).

We developed and used a standardized checklist to ensure that study protocol was followed. Each researcher who dropped a letter completed this checklist for every target vehicle used in the study. All letters were placed according to protocol.

Assigning Conditions. Each of the 24 locations received eight letters, two identical letters for each of the four study conditions. In this way it was possible to guard against systematic biases in the condition represented by the particular letter being dropped at a specific location. (For example, more male-medical letters might be dropped at a higher SES part of the city, which could alter the return rate for this condition.) Four letters were distributed at four different drop times, two letters during cycle one and two letters during cycle two, for both drop days. During the first drop day, all letters had a single, 39-cent stamp; during the second drop day, all letters had a 37-cent and a two-cent stamp.

A Latin square design determined the order of the drop condition (Kirk, 1968). As Kirk notes, "Theoretically, an experimenter who plans to use a Latin square design should randomly select a square from the population of all possible squares of the proper dimension" (Kirk, 1968, p. 154). Using the procedures described by Kirk, we randomly selected one of the 576 possible $4 \times 4$ Latin squares in which each study condition occurs once and only once in each row and column.

Thus, we did not randomly allocate participants to the conditions of this study; instead, we randomly assigned one of four conditions to each of the participants who picked up a letter. 


\section{KERN AND YEATON}

Hence, if each capital letter represents one of the randomly assigned conditions of the study, the Latin square we chose was:

C B D A

D A B C

B C A D

A D C B

More precisely, on the first drop (which corresponds to the first row of the design), all six locations in the first part of the city received condition $\mathrm{C}$; all six locations in a second part of the city received condition $\mathrm{B}$, and so forth, continuing across the row. On the second drop (which corresponds to the second row of the design), all six locations in the same first quadrant now received a different condition, condition $\mathrm{D}$; all six locations in the second quadrant of the city also received a new condition, condition A; etc. The last two rows represent the two drops during the second day in which letters were distributed. In summary, each of four city quadrants (and six locations within each quadrant) received every condition, but on two different days, on four different drop occasions, in a random order dictated by the Latin square design.

Sub-Study Replication. The design of this study allowed us to analyze the return rates of letters dropped in Cycle 1 of each weekend combined (row one and row three in Figure 1) and the return rates of letters dropped in both Cycle 2's (row two and row four of Figure 1). This approach, however, sacrificed the Latin square feature of the research design.

\section{Data Analytic Strategy}

Log-linear analysis is appropriate instead of Chi Square for a 2 × 2 × 2 table when data can be cross-classified in a number of ways (Agresti, 1996). Though, to our knowledge, previous LL studies have not used the log-linear approach, by framing the research as consisting of three factors, gender, medical profession, and return (yes/no), we could examine the pattern of returns within gender and profession as a two-way interaction. Otherwise, the log-linear approach is quite similar to a Chi square analysis, the tactic favored by previous LL researchers, with its degrees of freedom and appropriate sampling distribution (Lowrey, 2009).

Pilot Study. A pilot study used six letters in each of the cells of a 2 x 2 design (number of returned letters in each cell, with gender crossed by profession). The overall return rate was $54.2 \%$ (13/24); the proportion of letters returned in the medical condition was $66.7 \%(8 / 12)$ and $41.7 \%(5 / 12)$ in the chiropractic condition. The proportion in the group with male return addresses was $41.7 \%(5 / 12)$ and $66.7 \%(8 / 12)$ in the group with female return addresses.

Using the percentages produced in the pilot study, we planned to use 48 participants in each of four conditions of the formal study ( $n=192$ participants). A one-tailed test of significance with $\alpha=.05$, with $\mathrm{n}=96$ for each of the two major contrasts (male vs. female, medicine vs. chiropractic) produced a statistical power of a test of the difference between proportions of .92 (Cohen, 1988).

Dependent Variables. The proportion of letters that was returned (number returned/number sent) within 21 days from the date of drop served as the primary dependent variable. (In the pilot study all returned letters were returned within the first week after they were dropped.) We also determined the percent of letters that had been opened in each of the four conditions. 


\section{REFINING LOST LETTER METHODS}

\section{Results}

A log-linear analysis of the $2 \times 2 \times 2$ table (male/female by medical/chiropractic profession by return/not return) revealed no significant difference in the return rates of lost letters $\left(\mathrm{G}^{2}=\right.$ $.18, \mathrm{df}=4, \mathrm{p}>.05)$. An additional log-linear analysis of the data in Table 1 detected no significant interaction between gender and profession $\left(\mathrm{G}^{2}=0.0, \mathrm{df}=1, \mathrm{p}>.05\right)$. Both authors examined all returned letters and agreed that none had been opened.

\section{Table 1}

Distribution of returned letters by gender and health profession

\begin{tabular}{lcc} 
& & \\
\cline { 2 - 3 } & Medical & Profession \\
\hline Gender & Returned (not returned) & Returned (not returned) \\
\hline Male & $32(16)$ & $30(18)$ \\
Female & $31(17)$ & $31(17)$
\end{tabular}

Note. Each cell represents the number of returned letters of 48 lost letters (study $n=196$ ). Cell entries in parentheses represent the number of non-returned letters. $p>.05$, for both main effect $(\mathrm{df}=4)$ and interaction $(\mathrm{df}=1)$, using log-linear analysis (see text).

The design of this study allowed us to treat the larger study $(n=192)$ as if we had conducted two, replicate sub-studies of 96 lost letters. Thus, for a given weekend day, each of the two cycles resulted in 48 lost letters (in total, $n=96$ for Cycle 1 and $n=96$ for Cycle 2). We then aggregated rates by Cycle, combining results in the two, Cycle 1 lost letters and the results in the two, Cycle 2 lost letters. Within each cycle pair, further examination of the relation between gender and health orientation showed non-significant main effects $\left(\mathrm{G}^{2}=.38, \mathrm{df}=4, \mathrm{p}>.05\right.$, for Cycle $1 ; \mathrm{G}^{2}$ $=.36, \mathrm{df}=4, \mathrm{p}>.05$, for Cycle 2$)$ and interactions $\left(\mathrm{G}^{2}=.19, \mathrm{df}=1, \mathrm{p}>.05\right.$, for Cycle $1 ; \mathrm{G}^{2}=.18$, $\mathrm{df}=1, \mathrm{p}>.05$, for Cycle 2). (See Table 2) The pattern of returned letters between these two, $2 \mathrm{x}$ 2 sub-tables within Table 2 was similar.

Table 2

Distribution of returned letters by gender and health profession: Cycle 1 and Cycle 2 represent replicate sub-studies

\begin{tabular}{clcc}
\hline & & \multicolumn{2}{c}{ Profession } \\
\cline { 3 - 4 } Cycle 1 & Gender & Medical & Chiropractic \\
& Male & $17(7)$ & $16(8)$ \\
& Female & $16(8)$ & $15(9)$ \\
\hline Cycle 2 & & & $14(10)$ \\
& Male & $15(9)$ & $16(8)$ \\
& Female & $15(9)$ &
\end{tabular}

Note. Each cell in each cycle represents the number of returned letters of 24 lost letters. Cell entries in parentheses represent the number of non-returned letters. $p>.05$, for each Cycle, for both main effects $(\mathrm{df}=4)$ and interactions $(\mathrm{df}=1)$, using log-linear analysis (see text). 


\section{KERN AND YEATON}

\section{Discussion}

Despite previous evidence suggesting systematic patterns of difference, we did not find a significant difference between attitudes toward traditional and alternative medicine. Nor did we find that females were more likely to have their letters returned than males. In addition, there was no significant interaction between gender and medical profession (there were no differential rates of return for females in the chiropractic condition and males in the medical condition versus males in the chiropractic condition and females in the medical condition). Thus, at least in this one city, attitude toward the two kinds of medical professions seemed to be similar if not identical.

\section{Quality of Methods vs. Previous Studies}

The study applies a number of methodological embellishments to previous implementations of the LL approach. Thus, it is implausible that the no-difference findings are attributable to inadequate study methodology. In addition, the fact that the design incorporated a systematic replication, one which produced a substantially similar no-difference pattern, lends further credence to its conclusions (though the replication no longer used the Latin square design). Among others, Hill (1965) argued quite compellingly that a consistent pattern of findings enables one to reach more definitive conclusions. Finally, the pilot study power analysis indicated the present sample size was sufficient to detect reasonable-sized effects. However, if the true differences between conditions are of the magnitude of a few percent, and our work has successfully estimated these small differences, statistically significant comparisons will require a huge sample size.

Past studies using LL techniques have not directly addressed the question taken up in this study. Thus, we are not able to directly compare our findings with those in other research. However, and we regard this as a substantial "however," given the methodological deficiencies in the LL literature, there is a strong, logical case that some, previously published LL studies have reached spurious conclusions.

Non-random choice of study locations, study conditions too conveniently paired with drop sites, the fact that all study locations did not receive all study conditions, and the non-random order in which conditions were administered to sites together conspire to give reason to question the veracity of previous study results. For future efforts, it is imperative that all study conditions be given to all SES areas of a city and that each study condition is delivered to a location with a given SES. Minimally, a generalization to the particular city chosen will require that all city areas be represented and that within these areas, each adult resident has a reasonably equal chance of being included in the study.

Possible Inferential Limitations. The rates of return found in this study were middling compared to other LL studies (e.g., 55\% for automobiles in Milgram et al. [1965] and 88\% for Wicker [1969]). Indeed, rates were not so high as to introduce a ceiling effect (still higher rates of return were easily possible in all of our study conditions). These modestly high rates may be due to targeting lost letters to automobiles and their owners if auto owners are generally more responsible and more likely to return letters. Furthermore, we did not systematically identify mailboxes or post offices near the drop locations chosen here, but it is possible that proximity to these services had some influence on return rates. Like previous LL studies, we did not observe finders of lost letters, so we do not know the demographic characteristics (e.g., gender) of the potential helper. Of course, none of these factors was differentially present in the medical profession or gender conditions of this study. 


\section{REFINING LOST LETTER METHODS}

The chosen city was quite diverse in its SES and demographics, and is not an atypical medium sized-city. But, to the degree that other cities differ from the chosen city in salient predictors of return rates, we would expect study results to differ. Studies that sample from nonbusiness locations might also yield different findings.

Perhaps the relatively minimal effort required to mail a letter offers a partial explanation for why the return rates were substantially the same for males and females. With greater response cost on helping, females may have had more letters returned to them than males. In addition, there is consistent evidence for an increasingly greater acceptance of alternatives to traditional medicine by consumers (Kessler et al., 2001). So, in the context of this trend, the difference between attitudes toward chiropractic and traditional medicine may be decreasing (or, to be logically complete, favorable attitudes toward traditional medicine may be decreasing).

We have anecdotal evidence that finders of lost letters paid attention to the salient elements on envelopes. We received both phone calls and personal letters from individuals who found lost letters, and even discovered lost letters hand delivered to one author's mailbox (WHY). Finders often mentioned the names listed on the return address (Jessica and Jason) as well as the specific Institutes. We thanked each person who contacted us and ensured them that we would do our best to see that the respective Institute received the letter in a timely fashion.

Recapitulation and Conclusion. As demonstrated here, there was little evidence of differing attitudes toward chiropractic and traditional medicine. In addition, the research did not suggest differing attitudes toward gender roles within these two professions. Our findings, obtained in a context of several methodological refinements of a non-reactive measurement, are consistent with the growing acceptance of complementary and alternative approaches to medicine.

\section{Author Note}

The authors wish to thank Will Cummings and Kimberly Reid for their assistance in both planning and conducting this research. We are similarly indebted to Arwen Smith and Andrew Watkins for their help during the pilot phase of the study. The following colleagues provided thoughtful feedback on earlier drafts of the paper: Jake Jacobs, Michael Menke, Brian Moss, and

Riva Tukachinsky. Inquiries may be addressed to William Yeaton, E-mail: bill.yeaton@yahoo.com.

\section{References}

Agresti, A. (1996). An introduction to categorical data analysis. New York: John Wiley \& Sons.

Baumeister, R. F., Vohs, K. D., \& Funder, D. C. (2007). Psychology as the science of self-reports and finger movements. Perspectives on Psychological Science, 2, 396-403.

Beard, E. L. (2004). Consumer trust in healthcare organizations is waning. How will 21st century leaders bridge the gap? Nursing Administration Quarterly, 28, 99-104.

Berkowitz, W. R. (1970). Spectator responses at public war demonstrations. Journal of Personality and Social Psychology, 14, 305-311.

Bushman, B. J., \& Bonacci, A. M. (2004). You've got mail: Using e-mail to examine the effect of prejudiced attitudes on discrimination against Arabs. J ournal of Experimental Social Psychology, 40, 753-759.

Cohen, J. (1988). Statistical power analysis for the behavioral sciences. (2nd ed.) Hillsdale, NJ: Lawrence Erlbaum Associates.

Conboy, L., Kaptchuk, T. J., Eisenberg, D. M., Gottlieb, B., \& Acevedo-Garcia, D. (2007). The relationship between social factors and attitudes toward conventional and CAM practitioners. Complementary therapies in clinical practice, 13, 146-157. 


\section{KERN AND YEATON}

Eagly, A. H., \& Crowley, M. (1986). Gender and helping behavior: A meta-analytic review of the social psychological literature. Psychological Bulletin, 100, 283-308.

Eccles, J. S. (1986). Gender-roles and women's achievement. Educational Researcher, 15, 15-19.

Eisenberg, D. M, Kessler, R. C., Foster, C., Norlock, F. E., Calkins, D. R., \& Delbanco, T. L. (1993). Unconventional medicine in the United States-Prevalence, costs, and patterns of use. New England J ournal of Medicine, 328, 246-252.

Freedman, D, Pisani, R., \& Purves, R. (1998). Statistics (3rd ed.). New York: W.W. Norton.

Gould, S. J. (2005). Consumer attitudes toward health and health care: A differential perspective. J ournal of Consumer Affairs, 22, 96-118.

Hill, A. B. (1965). The environment and disease: Association or causation. Proceedings of the Royal Society of Medicine, 58, 295-300.

Kessler, R. C., Davis, R. B., Foster, D. F., Van Rompay, M. I., Walters, E. E., Wilkey, S. A., Kaptchuk, T. J., \& Eisenberg, D. M. (2001). Long-term trends in the use of complementary and alternative medical therapies in the United States. Annuls of Internal Medicine, 135, 262-268.

Kirk, R. E. (1968). Experimental design: Procedures for the behavioral sciences. Monterey, CA: BrooksCole.

Kunz, P. R., \& Fernquist R. M. (1989). Opinions on abortion as measured by the lost-letter technique. Psychological Reports, 65, 1343-1346.

Lawrence, D. J., \& Meeker, W. C. (2007). Chiropractic and CAM utilization: A descriptive review. Chiropractic \& Osteopathy, 15, doi:10-11-1186/1746-1340-15-2.

Lowrey, R. (2009). Log-linear analysis for a 2x2x2 table of cross-categorized frequency data. VassarStats. Retrieved April 2, 2009, from Vassar College Web site http://faculty.vassar.edu/lowry/log.html.

Matus, R. (2004). Chiropractic school angers FSU professors: Some threaten to resign over the proposed school. (2004, December 29). St. Petersburg Times. Retrieved April 7, 2009, from http://www.sptimes.com/2004/12/29/State/Chiropractic_school_a.shtml.

Milgram, S. (1977). The individual in a social world. New York: McGraw-Hill.

Milgram, S., Mann, L., \& Harter, S. (1965). The lost-letter technique: A tool of social research. Public Opinion Quarterly, 29, 437-438.

Orne, M. T. (1962). On the social psychology experiment: With particular reference to demand characteristics and their implications. American Psychologist, 17, 776-783.

Shadish, W. R, \& Cook, T. D. (2009). The renaissance of field experimentation in evaluating interventions. Annual Review of Psychology, 60, 607-629.

Shadish, W. R., Cook, T. D., \& Campbell, D. T. (2002). Experimental and quasi-experimental designs for generalized causal inference. Boston: Houghton Mifflin.

Simmons, C. H., \& Zumpf C. (1983). The lost letter technique revisited. J ournal of Applied Psychology, $13,510-514$.

Webb, E., Campbell D., Schwartz R., \& Sechrest L. (1966). Unobtrusive measures: Nonreactive measures in the social sciences. Boston: Houghton Mifflin.

Wicker, A. W. (1969). A failure to replicate the lost-letter technique. Public Opinion Quarterly, 33, 260262. 